\begin{abstract}
M. Ettouney
R. Daddazio

Weidlinger Associates, Inc. Applied Science Division 333 Seventh Avenue New York, NY 10001

F. DiMaggio Columbia University, Department of Civil Engineering and Engineering Mechanics New York, NY 10027

\section{Specification of Modal Participation Factors in Acoustic Scattering Problems}

Using the wet modes of a submerged structure as expansion functions, it is shown how the scattered far-field pressures induced by an incident harmonic wave may be modified in a prescribed manner. This is done by applying forces directly to the structural surface, which superposes a radiated pressure field on the scattered one. By appropriate choice of the applied nodal forces, modal participation factors associated with any combination of mode shapes may be controlled. The method is illustrated by a numerical example. (c) 1993 John Wiley \& Sons, Inc.
\end{abstract}

\section{INTRODUCTION}

In earlier articles [Ettouney, Daddazio, and DiMaggio, 1992; Daddazio, Ettouney, and Abboud, 1992], the analysis of coupled acoustic radiation and scattering problems based on expansions in the wet modes of the submerged structure was described, and the coding of a program called WETMODE to implement the procedure announced.

In this article, it is shown how specific wet mode contributions to far-field scattered pressures induced by an incident harmonic wave may be modified in a systematic way by superposing an appropriate radiation loading.

\section{COUPLED RADIATION AND SCATTERING PROBLEM}

In Fig. 1, $S$ denotes the interface between a submerged shell and the fluid surrounding it, often referred to as the wet surface. The shell may be acted upon both by forces applied directly to its interior surface and an incident wave exerting forces from both its pressure and velocity fields. Although the pressure induced in the fluid by the surface forces is often called radiated and that by the incident wave is called scattered, they may be treated in a unified manner.

\section{HARMONIC EXCITATION AND SURFACE RESPONSE}

If both excitations are harmonic with driving frequency $\Omega$, the normal surface displacements at the $m$ nodes $j$ of the discretized interface $S$ may be written in exponential form, with $i=\sqrt{-}$, as

$$
w_{j}=W_{j} e^{i \Omega t} \quad j=1,2 \cdots m
$$

in which the $W_{j}$ are complex amplitudes whose dependence on frequency has been suppressed. 


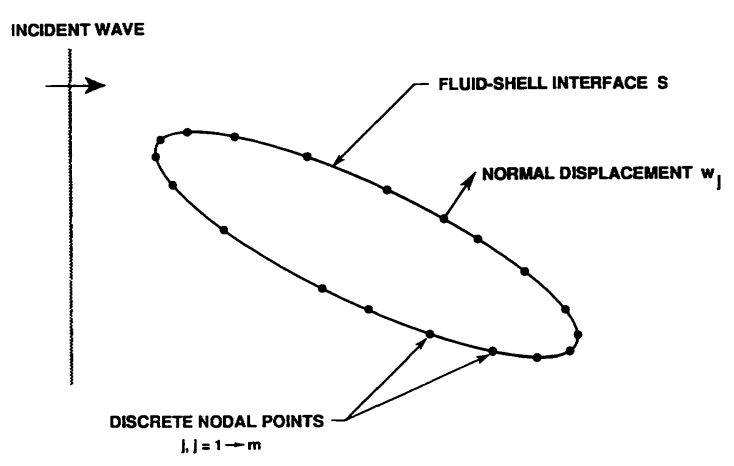

FIGURE 1 Description of the problem.

In matrix form, Eq. (1) becomes

$$
[w]=[W] e^{i \Omega t}
$$

in which $[w]$ and $[W]$ are $m \times 1$ column matrices or vectors and the real part of the right-hand sides of Eqs. (1) and (2) is to be understood.

If $\left[F_{r}\right]$ denotes the forces associated with radiation and $\left[F_{s}\right]$ those causing scattering, the equation of motion of this shell may be written as

$$
\left[K_{T}\right][W]=\left[F_{r}\right]+\left[F_{s}\right]
$$

in which $\left[K_{T}\right]$, the total shell impedance is the sum of the dynamic stiffness of the shell in vacuo and the interactive fluid impedance.

\section{WET MODES AND SURFACE PARTICIPATION FACTORS}

Let $\left[W_{k}\right], k=0 \rightarrow m-1$ denote the wet modes [Ettouney et al., 1992] associated with $S$. Then

$$
[W]=\sum_{k=0}^{m-1} \lambda_{k}\left[W_{k}\right]=\left[\Phi_{W}\right][\Lambda]
$$

in which $\left[\Phi_{W}\right]$ is the $m \times m$ augmented wet-mode displacement matrix formed from the column $\left[W_{k}\right]$ and $[\Lambda]$ is the $m \times 1$ vector of surface participation factors $\Lambda_{k}$.

Substituting (4) into (3), and taking the inverse,

$$
[\Lambda]=\left\{\left[K_{T}\right]\left[\Phi_{W}\right]\right\}^{-1}\left\{\left[F_{r}\right]+\left[F_{s}\right]\right\}
$$

that may be written as

$$
[\Lambda]=\left[\Lambda_{r}\right]+\left[\Lambda_{s}\right]
$$

In Eq. (6), $\left[\Lambda_{r}\right]$ is the matrix of surface participation factors for applied forces causing radiation and $\left[\Lambda_{s}\right]$ that corresponding to the forces resulting from the incident pressure causing scattering. They may be expressed in terms of these forces by means of Eq. (5) as

$$
\begin{aligned}
{\left[\Lambda_{r}\right] } & =[C]\left[F_{r}\right] \\
{\left[\Lambda_{s}\right] } & =[C]\left[F_{s}\right] \\
{[C] } & =\left\{\left[K_{T}\right]\left[\Phi_{W}\right]\right\}^{-1} .
\end{aligned}
$$

\section{FAR-FIELD PRESSURE MODE PARTICIPATION FACTORS}

The pressure in the far field may be written as [Ettouney et al., 1992]

$$
\left[p_{f}(\vec{x})\right]=\left[\Phi_{f}(\vec{x})\right]\left[\Lambda_{r}+\Lambda_{s}+\Lambda_{\infty}\right] .
$$

In Eq. (10), the wet-mode far-field pressure matrix

$$
\left.\left[\Phi_{f}(\vec{x})\right]=[E(\vec{x})]^{T}\left(-i \Omega_{W}\right]\right)
$$

[E( $\vec{x})]$ an $m \times 1$ vector function that relates farfield pressure to relative fluid velocity on $S$, may be calculated by standard methods described elsewhere [Abboud; Daddazio, Ettouney, Ranlet, and Smilowitz, 1992] and

$$
\left[\Lambda_{\infty}\right]=\left[\Phi_{W}\right]^{-1}\left[-\frac{i}{\Omega} V_{I}\right]
$$

in which $\left[V_{I}\right]$ is the incident velocity vector, is the correction to the surface participation factors due to rigid body scattering.

Letting

$$
\left[\Lambda_{f s}\right]=\left[\Lambda_{s}\right]+\left[\Lambda_{\infty}\right]
$$

denote the far-field scattering participation matrix, Eq. (10) may be written as

$$
\left[p_{f}(\vec{x})\right]=\left[\Phi_{f}(\vec{x})\right]\left\{\left[\Lambda_{r}\right]+\left[\Lambda_{f s}\right]\right\}
$$

or

$$
\left[p_{f}(\vec{x})\right]=\left[p_{r}(\vec{x})\right]+\left[p_{f s}(\vec{x})\right]
$$

or

$$
\left[p_{f}(\vec{x})\right]=\left[\Phi_{f}(\vec{x})\right]\left[\Lambda_{f}\right]
$$

in which

$$
\left[\Lambda_{f}\right]=\left[\Lambda_{r}\right]+\left[\Lambda_{f s}\right] .
$$




\section{FILTERING OF SCATTERED FAR-FIELD PRESSURES}

If an incident wave with known pressure and velocity field is prescribed, the participation factors $\left[\Lambda_{s}\right]$ and $\left[\Lambda_{\infty}\right]$ of Eqs. (8) and (12), and their sum of Eq. (13) are specified. This defines the scattered far-field pressure $\left[p_{f s}(\vec{x})\right]$ of Eq. (15), that may, however, be modified by a radiated pressure $\left[p_{r}(\vec{x})\right]$ to produce a resultant far-field pressure $\left[p_{f}(\vec{x})\right]$ as in Eq. (15). This radiated pressure may be generated by applying internal surface forces $\left[F_{r}\right]$, that add the participation factor $\left[\Lambda_{r}\right]$, of magnitudes determined from Eqs. (7) and (9) to Eq. (17).

Suppose, for example, it is desired to preassign fixed contributions of $l$ specific wet-mode shapes to the total far-field pressure for arbitrary incident waves.

Let the participation factors for far-field scattering be reordered so that the modes whose total contribution is to be fixed are located at the bottom of the vector. In partitioned form this may be denoted by

$$
\left[\Lambda_{f s}\right]=\left[\begin{array}{l}
{\left[\Lambda_{1 f s}\right]} \\
{\left[\Lambda_{2 f s}\right]}
\end{array}\right]
$$

in which the subvector $\left[\Lambda_{1 f s}\right]$ corresponding to modes not being monitored is of $m-l$, while that for the modes whose contribution is to be prescribed, is of order $l$.

Let internal forces $\left[F_{r}\right]$ be applied, inducing a radiated pressure, that, when added to the scattered far-field pressure, results in a total far-field pressure given by Eq. (15). The participation factor vector for this total pressure $\left[p_{f}(\vec{x})\right]$ can be partitioned in the same manner as that for $\left[p_{f s}(\vec{x})\right]$ in Eq. (18):

$$
\left[\Lambda_{f}\right]=\left[\begin{array}{l}
{\left[\Lambda_{1 f}\right]} \\
{\left[\Lambda_{2 f}\right]}
\end{array}\right] .
$$

If the resultant participation factors of the $l$ modes being monitored are arranged in an $l \times 1$ vector $\left[\bar{\Lambda}_{2 f}\right]$, the desired modification will be achieved by requiring

$$
\left[\Lambda_{2 f}\right]=\left[\bar{\Lambda}_{2 f}\right] .
$$

To determine the internal forces that must be applied to satisfy Eq. (20), Eq. (7) is first inverted to obtain

$$
\left[F_{r}\right]=[B]\left[\Lambda_{r}\right]
$$

in which, using Eq. (9),

$$
[B]=[C]^{-1}=\left[K_{T}\right]\left[\Phi_{W}\right] .
$$

Substituting Eq. (17) into Eq. (21) yields

$$
\left[F_{r}\right]=[B]\left\{\left[\Lambda_{f}\right]-\left[\Lambda_{f s}\right]\right\} .
$$

If $\left[F_{r}\right]$ and $[B]$ are partitioned to be compatible with the partitions of Eqs. (18) and (19), Eq. (23) becomes

$\left[\begin{array}{l}{\left[F_{1 r}\right]} \\ {\left[F_{2 r}\right]}\end{array}\right]=\left[\begin{array}{ll}{\left[B_{11}\right]} & {\left[B_{12}\right]} \\ {\left[B_{21}\right]} & {\left[B_{22}\right]}\end{array}\right]\left[\begin{array}{ll}{\left[\Lambda_{1 f}\right]} & -\left[\Lambda_{1 f s}\right] \\ {\left[\Lambda_{2 f}\right]} & -\left[\Lambda_{2 f s}\right]\end{array}\right]$.

Let $\left[F_{1 r}\right]$ be an $m-l$ order subvector of known applied internal forces (usually to be taken as zero) and $\left[\bar{F}_{2 r}\right]$ be the $l \times 1$ vector required to satisfy Eq. (20). Then Eq. (24) becomes

$$
\left[\begin{array}{l}
{\left[F_{1 r}\right]} \\
{\left[\bar{F}_{2 r}\right]}
\end{array}\right]=\left[\begin{array}{ll}
{\left[B_{11}\right]} & {\left[B_{12}\right]} \\
{\left[B_{21}\right]} & {\left[B_{22}\right]}
\end{array}\right]\left[\begin{array}{ll}
{\left[\left[\Lambda_{1 f}\right]\right.} & \left.-\left[\Lambda_{1 f s}\right]\right] \\
{\left[\left[\bar{\Lambda}_{2 f}\right]\right.} & \left.-\left[\Lambda_{2 f s}\right]\right]
\end{array}\right] .
$$

Solution of Eqs. (25) will yield the $l$ unknown forces in $\left[\bar{F}_{2 r}\right]$ that must be applied to satisfy Eq. (20) and the $m-1$ modified values in $\left[\Lambda_{1 f}\right]$ of the far-field participation factors not prescribed a priori.

\section{NUMERICAL EXAMPLE}

To illustrate the technique described above, consider the axisymmetric scattering problem of a plane harmonic wave impinging on a submerged spherical shell, as shown in Fig. 2. A detailed discussion can be found in [1]. The forward scatter pressures amplitudes, calculated as a function of driving frequency, are exhibited on the plot labeled "unfiltered" in Fig. 3. It was found that the resonances at 130,165 , and $190 \mathrm{~Hz}$ were primarily due to the $k=2, k=3$, and $k=4$ wet modes, respectively.

Assuming it is desired to suppress the resonances over the entire frequency range, this can be done by eliminating the contribution of the $k=2, k=3$, and $k=4$ for all $\Lambda$. Assuming further that we wish to leave all other wet modes unaffected and that internal forces can be applied at all wet nodes, no partitioning is necessary in 

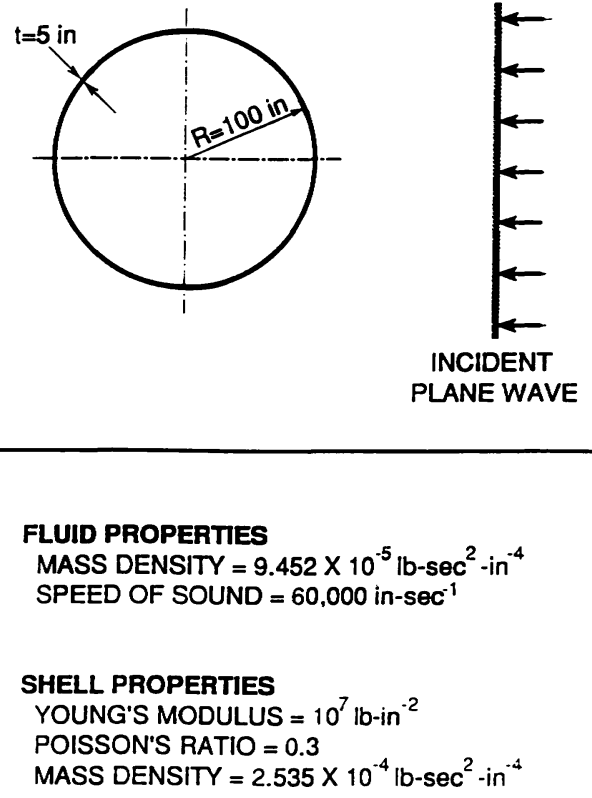

NOTE:

1 in $=0.0254 \mathrm{~m}$

$\mathrm{ilb}=0.453 \mathrm{~kg}$

FIGURE 2 Scattering from a thin elastic sphere.

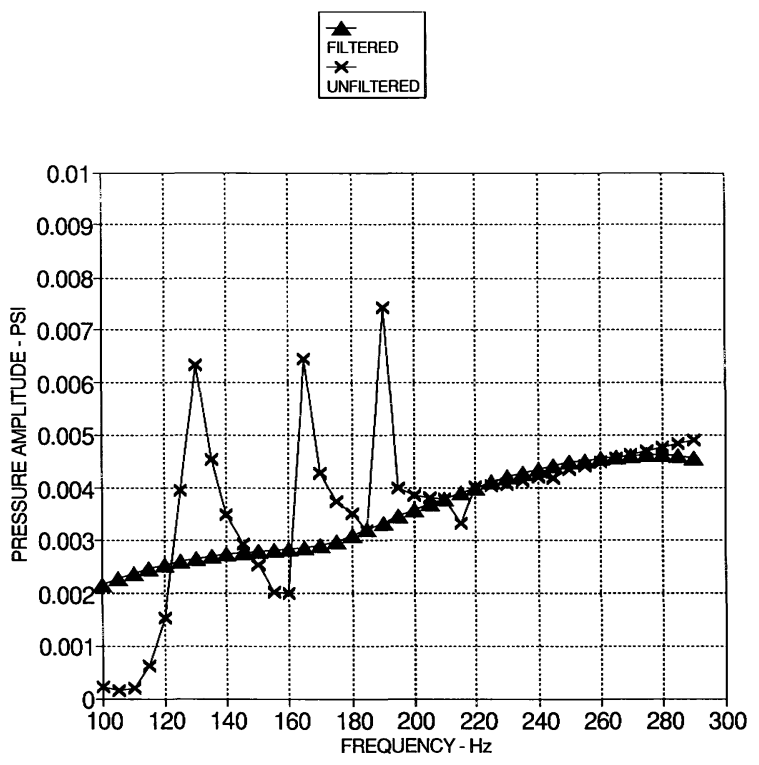

FIGURE 3 Filtered and unfiltered forward pressures.
Eq. (25) because $\left[F_{1 r}\right],\left[\Lambda_{1 f}\right]$ and $\left[\Lambda_{1 f s}\right]$ do not exist because

$$
l=m
$$

and

$$
\bar{\Lambda}_{2 f}=\left[\begin{array}{c}
\Lambda_{f s 0} \\
\Lambda_{f s 1} \\
0 \\
0 \\
0 \\
\Lambda_{f s 5} \\
\vdots \\
\Lambda_{f s l-1}
\end{array}\right]
$$

in which $\Lambda_{f s i}$ means the value of $\Lambda_{f s}$ corresponding to $k=i$. The filtered total pressure exhibited in Fig. 3 shows that the three unwanted model contributions have been eliminated.

\section{CONCLUSION}

A method has been presented to modify, in a systematic manner, the scattered pressure field from a submerged structure subjected to a harmonic incident wave. It is shown that a suitable superposition of radiation loading can result in the prescription of fixed wet-mode contributions to the far-field pressure.

A numerical example for a spherical shell and an incident plane wave illustrates how the method may be used for filtering far-field scattered pressures.

\section{REFERENCES}

Abboud, N. N., Daddazio, R., Ettouney, M. M., Ranlet, D., and Smilowitz, R., April 1992, "Acoustic Radiation/Scattering Response of a Linearly Elastic Shell of Revolution Containing Internal Structure-User's Manual for the WASCAT Code," Technical Report Number WA 9202, Weidlinger Associates, Inc., New York.

Daddazio, R. P., Ettouney, M. M., and Abboud, N. N., October 1992, "Wet Modes of Submerged Structures-Part 2: Applications," Transactions of the ASME, Journal of Vibrations and Acoustics, Vol. 114, pp. 440-448.

Ettouney, M. M., Daddazio, R. P., and DiMaggio, F. L., October 1992, "Wet Modes of Submerged Structures-Part 1: Theory," Transactions of the ASME, Journal of Vibrations and Acoustics, Vol. 114, pp. 433-439. 

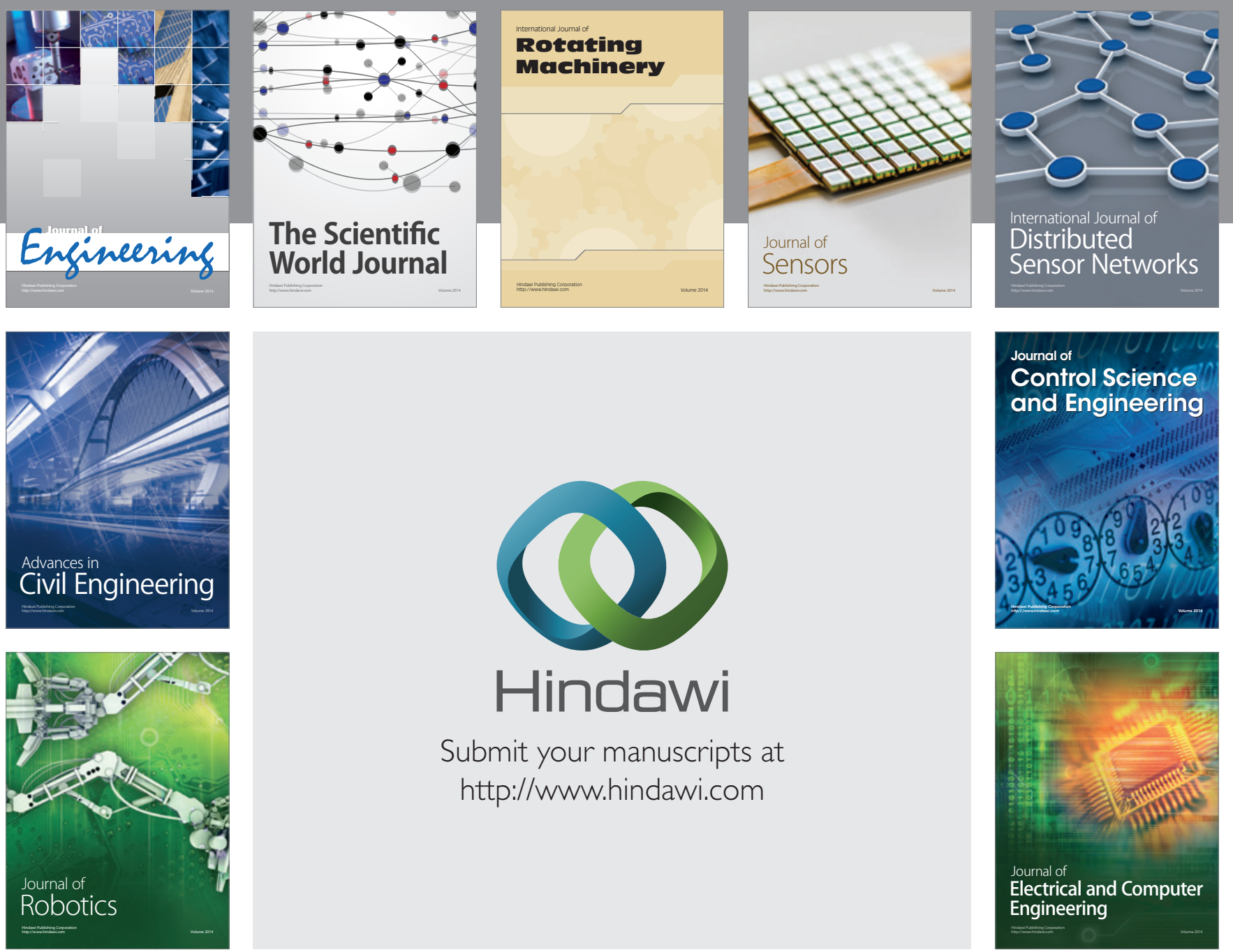

Submit your manuscripts at

http://www.hindawi.com
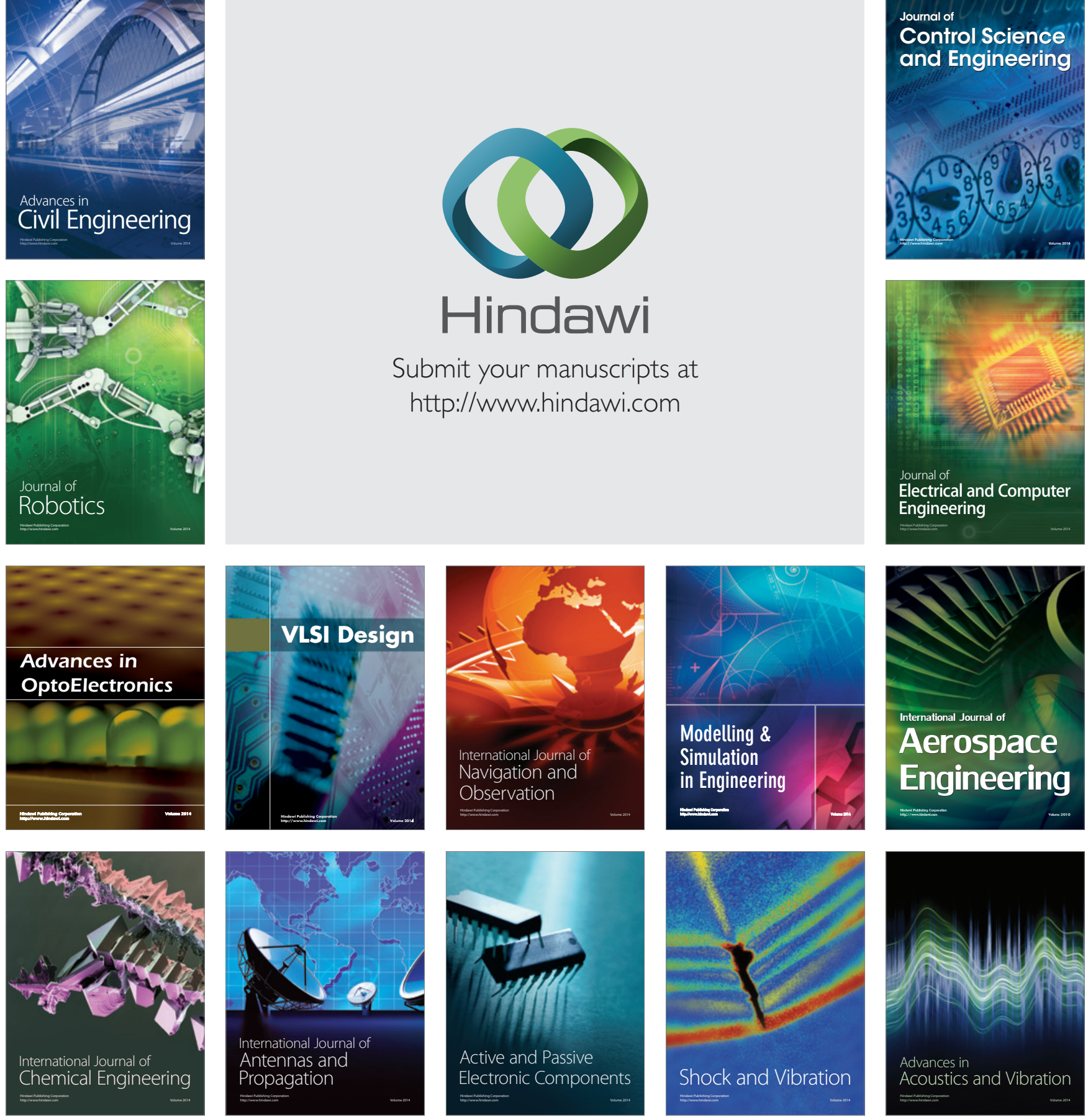